\title{
Prediction of autism spectrum disorder diagnosis using nonlinear measures of language-related EEG at 6 and 12 months
}

Fleming C. Peck

Boston Children's Hospital

Laurel J. Gabard-Durnam

Northeastern University

Carol L Wilkinson ( $\sim$ Carol.Wilkinson@childrens.harvard.edu )

Boston Children's Hospital https://orcid.org/0000-0002-4694-8564

William Bosl

Boston Children's Hospital

Helen Tager-Flusberg

Boston University

Charles A. Nelson

Boston Children's Hospital

Research

Keywords: EEG, Autism, Language Development, Machine Learning, Infant, Sensitive Period

Posted Date: April 21st, 2021

DOl: https://doi.org/10.21203/rs.3.rs-433976/v1

License: (9) (i) This work is licensed under a Creative Commons Attribution 4.0 International License. Read Full License

Version of Record: A version of this preprint was published at Journal of Neurodevelopmental Disorders on November 30th, 2021. See the published version at https://doi.org/10.1186/s11689-021-09405-x. 


\section{Abstract}

Background: Early identification of autism spectrum disorder (ASD) provides opportunity for early intervention and improved outcomes. Electroencephalography (EEG) use in infants has shown promise in predicting later ASD diagnoses and in identifying neural mechanisms underlying the disorder. Given the high co-morbidity with language impairment, we and others have speculated that infants who are later diagnosed with ASD have altered language learning, including phoneme discrimination. Phoneme learning occurs rapidly in infancy, so altered neural substrates either during or after the first year may serve as early, accurate indicators of later autism diagnosis.

Methods: Using longitudinal EEG data collected during a passive phoneme task in infants with high familial risk for ASD, we compared the predictive accuracy of a combination of feature selection and machine learning models at 6 months (during phoneme learning) versus 12 months (after phoneme learning), and identified a single model with strong predictive accuracy $(100 \%)$ for both ages. Samples at both ages were matched in size and diagnoses ( $n=14$ with later $A S D ; n=40$ without $A S D)$. Features included a combination of power and nonlinear measures across 10-20 electrodes and 6 frequency bands. Predictive features at each age were compared both by feature characteristics and EEG scalp location.

Results: Using a combination Pearson correlation feature selection and support vector machine classifier $100 \%$ predictive diagnostic accuracy was observed at both 6 and 12 months. Predictive features differed between the models trained on 6-versus 12- month data. At 6-months, predictive features were biased to measures from central electrodes, power measures, and measures in the alpha range. At 12-months, predictive features were more distributed between power and nonlinear measures, and biased toward measures in the beta range.

Conclusions: These results demonstrate that speech processing EEG measures can facilitate earlier identification of ASD but emphasize the need for age-specific predictive models with large sample sizes to develop clinically relevant classification algorithms.

\section{Background}

The past decade has witnessed a dramatic increase in the prevalence of autism spectrum disorder (ASD), a neurodevelopmental disorder characterized by social communication and repetitive and restrictive behaviors (1). The CDC estimates that one in 54 children has an ASD diagnosis (2), up from the one in 88 prevalence reported about a decade ago (2). Currently, ASD is diagnosed using behavioral measures, so a diagnosis cannot be made until toddlerhood or later when behavioral symptoms are reliably observable (3). However, there is strong support for the assertion that early intervention leads to better intellectual and behavioral outcomes $(4,5)$. Therefore, a central focus for the field has been to develop identification tools using biological markers to facilitate earlier detection and subsequent intervention of ASD. 
Neuroimaging measures provide strong candidate tools for early identification as they can be obtained from the newborn period onwards. For example, several recent studies have used magnetic resonance imaging (MRI) data collected in infancy to predict ASD diagnoses(6,7). However, MRI has several drawbacks, including expense and participant restrictions, making it a less feasible general screening tool. Electroencephalography (EEG), on the other hand, may prove to be a more scalable tool, given its low cost and ease of acquisition in awake and sleeping infants without participant restrictions. Moreover, EEG is known to be sensitive to brain-related changes in ASD before behavioral symptoms are observable (812). Initial efforts to predict ASD diagnoses using baseline (i.e. resting-state) EEG early in life have shown promise (13-16). However, diagnostic prediction using EEG recorded during tasks related to ASD symptoms has yet to be attempted and may outperform prior baseline EEG-based classification.

Language is frequently delayed or impaired in ASD (17-21), which may result from atypical peak synaptic sensitivity (22) and cortical excitatory and inhibitory imbalance (23) that disrupt neural circuits typically involved in language development. Therefore, focusing on the brain's electrical activity during language processing may facilitate improved diagnostic prediction accuracy relative to baseline conditions, and provide insights into the neurobiology of language processing deficits within ASD. Notably, EEG has been used to measure differences in language processing in children with ASD who are older than 12 months (24-26), suggesting EEG is sensitive to atypical neural processing of language stimuli in ASD.

Perceptual narrowing of phoneme discrimination is a critical first stage in language acquisition $(26,27)$ and may be processed differently in infants with ASD (28-30). That is, very young infants can discriminate between native and non-native phonemes better than adults, but they lose this ability over the first year of life as their phoneme perception is tuned to the language(s) experienced in daily life (31). Therefore, this study focused on the phoneme learning window over the first year of life as a potential source of early indicators of subsequent ASD diagnosis.

There were two overarching goals of the present study. First, we aimed to evaluate whether EEG data collected during a language phoneme task at either 6- or 12-months of age in infants with familial risk for ASD can accurately predict later ASD diagnosis. We utilized EEG data collected from high familial risk infant siblings as part of a prospective longitudinal study, where diagnosis of ASD was determined at 23 years of age. Though power analysis of EEG is most common, nonlinear measures can capture dynamical properties of the brain that power analysis is not able to quantify. Nonlinear measures of adult EEG have accurately classified other clinical conditions, including depression (32-34), schizophrenia (35-37), and epilepsy (38-40). Our lab previously found that these measures computed from restingstate EEG are useful in predicting ASD outcome (15), and we now aim to improve predictive capacity by evaluating these measures on task-related data. Second, given that expected perceptual narrowing of phoneme discrimination occurs between 6 and 12 months of age, we aimed to compare the EEG features most predictive of diagnosis and determine whether there are developmental differences in which features are most important during versus after the language phoneme learning period. 


\section{Methods}

\section{Study design and participant demographics}

Participants were recruited to Boston Children's Hospital and Boston University to participate in a longitudinal study of infant siblings of children with ASD. Institutional review board approval was obtained from Boston University and Boston Children's Hospital (\# X06-08-0374). All infants had a gestational age greater than 36 week, no history of seizures, prenatal drug exposure, hearing impairment, or known genetic mutation involved in neurodevelopment. Infants were designated high risk (HR) for ASD based on the confirmed ASD clinical diagnosis of an older sibling. $104 \mathrm{HR}$ infants were enrolled in the longitudinal study and ASD outcome was determined using the Autism Diagnostic Observation Schedule (ADOS) in conjunction with a clinical best estimate. For infants meeting criteria on the ADOS or coming within 3 points of clinical diagnosis cutoffs, a Licensed Clinical Psychologist reviewed scores and video recordings and provided a best estimate clinical judgment of ASD diagnosis.

Three sets of EEG data were evaluated in predictive models: a 6-month sample, full 12-month sample, and matching 12-month sample. At 6-months, EEGs from 54 HR infants were analyzed (14 HR-ASD, 40 HR-noASD). A "matching" 12-month dataset was curated to assess longitudinal changes in ASD prediction at the different ages. $13 \mathrm{HR}-\mathrm{ASD}$ and $24 \mathrm{HR}$-noASD participants contributed data at both timepoints. The single HR-ASD participant in the 6-month cohort who did not contribute data at 12 months was replaced by an HR-ASD, demographically matched participant. All 40 HR-noASD 12-month samples were included in the matched dataset, resulting in the same sample size as that of the 6-month dataset. Additional prediction analyses were performed on all EEGs collected at 12 months; this larger sample included 67 HR infants (27 HR-ASD, 40 HR-noASD). Demographic and data quality information of each outcome group is presented in Table 1. Fisher's exact test was used to evaluate differences of demographic information between groups. The 12-month HR-ASD group had significantly lower mean maternal education than the HR-noASD group $(p=0.004)$. No other significant demographic differences were observed.

\section{Behavioral assessments}

The Mullen Scales for Early Learning (MSEL) and ADOS were administered at each data collection visit of the longitudinal study, including 6 and 12 months. The MSEL provides an index of ability in domains including language, cognition, and motor development.

\section{EEG paradigm}

The phoneme speech task consisted of a stream of consonant-vowel stimuli, presented using a doubleoddball paradigm. Three phonemes were presented based on a probability of selection: the English standard "/da/" presented $80 \%$ of the time, the native deviant "/ta/" presented $10 \%$ of the time, and the non-native deviant "da/" presented $10 \%$ of the time. A maximum of 600 stimuli were presented with a variable interstimulus period of minimum $700 \mathrm{~ms}(29)$. 


\section{EEG data acquisition and processing}

EEG data were acquired in a dimly lit, sound-attenuated, electrically-shielded room. A research assistant was present in the room to ensure that the infant remained calm and still during the language paradigm by blowing bubbles or presenting toys if the infant became distracted or fussy. Assistants did not engage in social interaction with the infant during task completion. EEG data were collected with either a 64channel Geodesic Sensor Nets or a 128-channel Hydrocel Geodesic Sensor Net (Electrical Geodesics, Inc (EGI), Eugene, OR, USA), using a $0.1 \mathrm{~Hz}$ high-pass analog filter and online rereferencing to the vertex (channel $\mathrm{Cz}$ ) through NetStation software (EGI, Eugene, OR, USA). Impedances were kept below $100 \mathrm{~K} \Omega$ in accordance with the connected DC-coupled amplifier (Net Amps 200 or Net Amps 300, Electrical Geodesics, Inc.). Data were sampled at either 250 or $500 \mathrm{~Hz}$.

EEG data were exported from NetStation to MATLAB format (R2017A). Files were batch processed using the Harvard Automated Processing Pipeline for EEG (HAPPE) within the Batch Electroencephalography Automated Processing Platform (BEAPP) software $(41,42)$.

Data were $1 \mathrm{~Hz}$ digital high-pass and $100 \mathrm{~Hz}$ low-pass filtered were applied to each file, downsampled to $250 \mathrm{~Hz}$ (if needed), and run through the HAPPE module using a spatially distributed subset of channels (Fig. 1). HAPPE artifact removal steps included bad channel identification, electrical line noise removal, artifact removal through wavelet-enhanced ICA with automated component rejection via the Multiple Artifact Rejection Algorithm $(43,45)$. Bad channels were then interpolated and EEG data were rereferenced to the average reference and mean signal detrended.

\section{EEG data decomposition}

The middle 20 seconds of the longest stretch of consecutive standard phoneme (English 'da') presentations in each file were selected for analysis to maximize the number of participants included while ensuring nonlinear measures could be calculated with fidelity. EEG data from 10-20 channel equivalents (18 channels) for each net type were then decomposed into frequency sub-bands using a discrete wavelet transform and a coarse-graining procedure (see Supplementary Materials for description).

\section{EEG measures}

11 different nonlinear measures and power were computed for each waveband from each of the 18 electrodes for each participant (Table 2).

\section{Feature Selection Strategies}

Across models, three distinct feature selection methods were evaluated to prevent model overfitting: selection based on (1) the features most correlated with autism outcomes (Pearson correlation coefficient), (2) the features with most significant $F$ ratio of mean square variances by group (F-test), and (3) the features selected using recursive feature elimination (RFE) with cross validation based on a linear support vector machine. Each selection method was restricted to selecting 20 features. 


\section{Classification strategies}

We evaluated multiple prediction models that varied in classification approach, including a support vector machine (SVM) with radial basis function, a Gaussian Naïve Bayes algorithm, linear discriminant analysis, and a $k$-nearest neighbors model where $k=7$, the square root of the sample size of the 6-month and matching 12-month datasets. All other models were trained using default parameters from the Python open source package scikit-learn.

Previous studies have found that including test samples in feature selection biases prediction accuracies (46-50), so we employed nested leave-one-out cross validation to evaluate the validity of model performance. Five metrics were used to evaluate model performance: accuracy, sensitivity, specificity, positive predictive value, and negative predictive value. Given the imbalanced nature of the sample, permutation testing was used to assess the significance of observed prediction accuracy. A null distribution of predictive accuracy was generated by repeating the following procedure over 1000 iterations: diagnostic labels were shuffled and cross validation analysis using features selected with true labels was performed.

\section{Characterizing Selected Features Across 6 and 12 months}

To determine the frequency of feature characteristics (i.e. channel, frequency band, measure) selected during the nested leave-one-out procedure (Fig. 1), the 20 features selected over each of the 54 iterations for 6-month and matching 12-month data sets were collated over the characteristic categories, summed, and then divided by the total iteration count. Significant differences between diagnostic groups were evaluated with a student's t-test with Bonferroni correction for multiple comparisons.

\section{Results}

\section{Autism prediction at 6 and 12 months}

Various prediction models drawing from three feature selection methods and four machine learning classifiers were evaluated for prediction accuracy of future autism diagnosis using either 6-month or 12month matched EEG datasets (each with 40 HR-noASD and 14 HR-ASD participants). The SVM classifier with features selected by the Pearson correlation ranking method achieved $100 \%$ diagnostic prediction accuracy for both ages while other classification attempts were more variable (Table S2). Predictive accuracy using true labels was significantly better than chance as determined by the null distribution generated with random labels for both timepoints (6-month z-score 7.35, $p<0.0001 ; 12$-month z-score 7.5, $p<0.0001$ ).

\section{Predictive features at 6 and 12 months}

The nature and spatial distribution of features selected in the successful 6- and 12-month predictive models were extracted in order to compare EEG features most predictive of 
ASD diagnosis either during or after perceptual narrowing of phoneme discrimination. Importantly, the same feature selection method and machine learning algorithm (Pearson correlation coefficient feature selection and an SVM classifier) achieved $100 \%$ predictive accuracy for both the 6-month and 12-month matched datasets, allowing for direct comparison of the features selected at each iteration of nested cross validation across the two ages.

Figure 2 shows the selection rates of features by channel, measure, and frequency. At 6 months, features were selected largely from central and left of the midline locations (Fig. 2A), and power was the most frequently selected measure (Fig. 2B). Five of the 12 measures (power, approximate entropy, Hurst exponent, Lempel-Ziv complexity, and permutation entropy were consistently selected across iterations. Within iterations, power was most frequently selected. Additionally, while almost all frequency bands were selected at each iteration, signal filtered to frequency ranges below $16 \mathrm{~Hz}$ were more frequently selected (Fig. 2C). At 12 months, selected channels changed most in the left hemisphere, with increased left-lateralization (i.e. shifts away from midline) and representation from especially dense frontal and temporo-parietal regions (Fig. 2D). Seven of the 12 measures were selected in at least $80 \%$ of the iterations, and half of the selected measures in each iteration were power or Lyapunov exponent computed at different wavebands and channels (Fig. 2E). While measures related to all frequency bands were consistently chosen across iterations, the average count per iteration of features related to the 15.6$31.2 \mathrm{~Hz}$ range (largely canonical Beta frequencies) was nearly double any other frequency range (Fig. 2F).

We next compared the mean value of each of the 20 most frequently selected features between ASD outcome groups (Table 3). After correcting for multiple comparisons, only approximate entropy computed from the $\mathrm{F} 3$ electrode in the delta range $(1-4 \mathrm{~Hz})$ was significantly different between the two groups at 6 month (HR-ASD: mean $0.815 \pm 0.08$; HR-noASD: mean $0.76 \pm 0.037 ; p<0.0025$ ). In contrast, at 12 months of age, the mean values measured for seven of the 20 features most commonly chosen across iterations were significantly different between groups such that HR-ASD infants had consistently higher values for each of these features than HR-noASD infants. Significant features across model iterations were also those that were most often chosen during feature selection (Fig. 2E): Lyapunov exponent, Hurst Exponent, sample entropy, and power. Lempel-Ziv complexity was the only measure selected in all iterations that was not significantly different between groups after Bonferroni correction.

\section{Autism prediction at 12 months: full sample}

We next evaluated the diagnosis prediction accuracy using all available 12-month participants and found a considerable decrease in almost all evaluation metrics was observed (Table S2), including the combination of Pearson correlation coefficient feature selection and SVM classifier which dropped from $100-7 \%$ accuracy when the sample was expanded. Only one of the 12 classification schemes-F-test feature selection with SVM algorithm-resulted in accuracy marginally above chance (61.2\%) at the severe expense of sensitivity (3.7\%), the measure evaluating the percentage of infants with ASD who were predicted correctly. 
Given this discrepancy, we assessed whether there were behavioral differences in the HR-ASD participants between full and matched 12-month samples that could provide possible explanation for the differences in brain-based classification accuracy (Table 4). We found that 12-month participants who also participated at the 6-month timepoint had significantly lower severity scores on the ADOS at 36 months compared to 12-month infants who only contributed data at the 12 month timepoint.

\section{Discussion}

This study evaluated multiple classification schemes to predict ASD diagnosis in high-familial risk infants using language-task EEG data. Specifically, EEG was evaluated at 6- or 12-months of age, timepoints that span a critical early language-learning period in development. $100 \%$ diagnostic classification accuracy was achieved using the Pearson correlation coefficient feature selection with the SVM classifier regardless of whether infants were within the critical period of language phoneme learning (6 months) or after (12 months). However, the features selected to achieve the $100 \%$ prediction rate differed between the two ages both in measure type and spatial distribution. Importantly, overall performance across models tested was highly variable and notably reduced when the sample demographics and size changed. Although we used robust statistical methods to limit overfitting in small samples, these constrained models may be unable to fit the variability present in ASD. The implications of these results in the search for early neuroimaging biomarkers of ASD diagnosis are discussed below.

\section{Developmental shift in predictive features}

The longitudinal nature of the study provides the opportunity to assess whether predictive EEG features change across the course of a language-learning critical period over the first year of life. Several differences were identified. At 6 months, power from frequencies below $16 \mathrm{~Hz}$ dominated as the most common measures selected, whereas at 12 months additional nonlinear measures were more consistently selected with a shift toward high frequencies, suggesting that nonlinear measures of high frequency signal better define binary ASD diagnostic outcomes based on language task EEG at 1 year of life. This trend is consistent with previous longitudinal studies that observe power differences between high- and low-risk infants emerging before 6 months but often dissipating by 12 months (50-53). While power was the most common feature type selected at 6-months, few features were statistically different between HR-noASD and HR-ASD groups at this age after correcting for multiple comparisons. In contrast, at 12 months, significant differences between outcome groups were identified in 7 of the 20 most common features (Table 3). This suggests that different classification strategies are utilized at the two ages, with a combination of different power features taken together at 6 months and independent contributions of a range of power and nonlinear features at 12 months.

We also observed differences between developmental time points topographically, with a shift leftwards from central-midline locations, including increased involvement of the temporal and lateral frontal cortex at 12 months (Fig. 2G). Power measures computed from the left-frontal F7 channel were particularly predictive as F7 power from all five frequency ranges were identified as 5 of the 20 most commonly 
selected features at 12 months (Table 3), and F7 power between $1-15.6 \mathrm{~Hz}$ was significant increased in the HR-ASD group compared to HR-noASD. This shift may indicate that infants who are later diagnosed with ASD show atypical language processing in the network of left-lateralized regions involved in healthy language perception by 12 months of age (47,54-57). Our EEG-related findings corroborate MRI findings of atypical activation related to passive auditory stimuli in adults and children with ASD over similar cortical areas $(46,58,59)$.

\section{Variable classification performance}

Our evaluation of machine learning models identified a single model with high accuracy for matched 6and 12-month data sets. However, the nested leave-one-out cross validation results varied greatly across different feature selection method and machine learning algorithm combinations. Performance variability may be attributed to the small size of the datasets, which risk overfitting or underfitting to the training data despite our efforts to minimize the dimensionality of the data before classification. Similarly sized studies using MRI data have previously demonstrated sensitivity lower than $90 \%$ with near-perfect specificity (60-62). These other studies only presented results from a single classification scheme, so the variability across classification schemes for MRI data is unknown.

The decrease in accuracy from $100 \%$ with the matched 12 -month dataset to $7 \%$ with the full 12 -month dataset using a SVM classifier suggests an inability to effectively separate the two diagnostic outcome classes after the HR-ASD group was expanded. Behavioral phenotypes of the HR-ASD infants added to complete the full 12-month analysis were more variable and severe than those who participated at both ages. It is possible that enrollment bias influenced the sample characteristics in that high-risk families enrolling at a later age may have had increased concerns about ASD related to observed symptoms. We postulate that the inclusion of more samples resulted in increased feature overlap across outcome classes, preventing accurate hyperboundary separation. Our 12-month results also suggest that more complex modeling will be required at this age to appropriately account for the full range of heterogeneity in ASD at the brain and behavioral levels. It is possible that classification with simpler classification approaches still perform well with an increased 6-month sample size if EEG measures tend to be less variable and better stratified between the ASD diagnostic outcomes at this younger age.

\section{The effect of heterogeneity and sample size on prediction}

Autism is a heterogenous disorder-its defining categories are broad and encompass a spectrum of symptom severity. Our observed decline in classification accuracy with increased sample size and important shifts in sample phenotypes (including ADOS severity) highlights factors that must be considered by the greater autism research community for future diagnosis prediction efforts. This withinstudy classification distinction serves as a case study of poor generalizability to a larger sample.

The HR-ASD infants who participated in both 6- and 12-month timepoints had significantly different ADOS severity scores at 36 months than the HR-ASD infants who joined the study at 12 months. On all five behavioral measures evaluated in Table 3, the 12-month-only HR-ASD group had higher ADOS scores and lower MSEL scores (corresponding to overall lower indexes of development). We hypothesize that the 
inclusion of a more severe and variable dataset reduced the accuracy of classification since we were predicting ASD as a binary diagnosis. Using resting-state EEG from the same infant-sibling dataset in this analysis, we have previously observed a similar lower accuracy in a 12-month sample, although a similar size sample at 9 months had high accuracy. As discussed in our previous paper, this may be a real neurodevelopmental trend reflecting the cross-over of neurodevelopmental trajectories of infants who do and do not go on to develop ASD $(14,15)$. Alternatively, this finding may correspond with previous metaanalyses of brain-disorder prediction field that have found decreasing accuracies reported as sample sizes-and, importantly, heterogeneity within the sample-increase (60-62). Overall, more sample data are needed in order to more completely represent the brain activity differences that arise in ASD, which would also permit the use of more complex models that may more appropriately account for the variability and complex associations between brain activity and diagnosis.

\section{Limitations and future directions}

We acknowledge several limitations of the current study in addition to the discussed challenges of sample size and heterogeneity. First, our focus on infants with familial risk of autism may not generalize to other ASD-risk groups or to the general population. Second, the specificity of our findings to ASD (versus other comorbid conditions) is unknown. Further testing across clinical populations (e.g., global developmental delay without ASD or isolated language delay) are needed to understand whether EEG could also be used to predict comorbidities with significant impact on functional outcomes. This study determines ASD outcome at age 3, which is appropriate for assessing ASD but not for many other developmental conditions that emerge across early childhood. Therefore, questions about comorbidity call for the extension of longitudinal studies to track participants beyond 3 years to capture a more complete clinical description of participants. Third, our sample was not diverse ethnically, racially, or in income level. Predictive analyses require not only large sample sizes but must include infants from diverse populations in order to improve clinical applicability to the general population. These results suggest that collaboration across samples is critical to moving forward in developing early predictive models.

Future studies of early predictive markers of ASD and other neurodevelopmental disorders need to be acutely aware of participant age, given the dramatic developmental changes in predictive feature profiles over the 6-month age window in the study. Moreover, given the variability of behavioral measures within the ASD outcome group, future studies should consider distinguishing different subpopulations of ASD grouped by biological presentations or phenotype profiles at the behavioral level as opposed to only binary diagnosis.

\section{Conclusions}

These results demonstrate that speech processing EEG measures may facilitate earlier identification of ASD. However, different nonlinear and power measures were predictive of ASD outcomes depending on developmental age with respect to early language learning. Overall, these findings emphasize the need for age-specific predictive models with large sample sizes. 


\section{List Of Abbreviations}

ADOS: Autism Diagnostic Observation Schedule

ASD: Autism Spectrum Disorder

BEAPP: Batch EEG Automated Processing Platform

EEG: Electroencephalography

EGI: Electrical Geodesics, Inc

HAPPE: Harvard Automated Preprocessing Pipeline for EEG

HR: High Risk

MARA: Multiple Artifact Rejection Algorithm

MRI: Magnetic Resonance Imaging

MSEL: Mullen Scales of Early Learning

SVM: Support Vector Machine

\section{Declarations}

Ethics approval and consent to participate: Institutional review board approval was obtained from Boston Children's Hospital (IRB\#X06-08-0374) prior to starting the study. Written, informed consent was obtained from all caregivers prior to their children's participation in the study.

Consent for publication: Not applicable.

Availability of data and material: Python and Jupyter Notebook scripts used for computation of nonlinear measures and machine learning analysis are available. The datasets of preprocessed nonlinear and power measures analyzed during the current study are available from the corresponding author on reasonable request.

Competing Interests: The authors declare that they have no conflicts of interest.

Funding: Support for this work was provided by: The National Institutes of Health (R01-DC010290 to HTF and CAN; R21 DC 08637 to HTF; 1T32MH112510 to CLW; 1K23DC017983), Autism Speaks (1323 to HTF), Simons Foundation (137186 to CAN) and the Princeton Fifty-Five Fund for Senior Thesis Research (FP). 
Author contributions: C.A.N. and H.T.F. designed the longitudinal study. L.G.-D. and C.L.W. processed the electrophysiological data. F.C.P., C.L.W., L.G.-D., and W.B. designed and carried out the analyses. F.C.P. and W.B. authored the code. F.C.P. drafted the paper, and all authors provided critical revisions.

Acknowledgments: We thank all of the families who participated in this study. We also thank Dr. Casey Lew-Williams for his thoughtful feedback and support of this project. Finally, we thank the entire ISP study team for their role in data collection and study coordination.

\section{References}

1. American Psychiatric Association. (2013): Diagnostic and Statistical Manual of Mental Disorders, 5th ed.

2. United States. Autism and Developmental Disabilities Monitoring Network Surveillance Year 2008 Principal Investigators. Morbidity Mortality Weekly Report: Surveillance Summaries. 2012;61:1-19.

3. Piven J, Elison JT, Zylka MJ. (2017, October 1): Toward a conceptual framework for early brain and behavior development in Autism. Molecular Psychiatry, Vol. 22. Nature Publishing Group, pp. 1-10.

4. Cohen H, Amerine-Dickens M, Smith T. (2006): Early intensive behavioral treatment: Replication of the UCLA model in a community setting. J Dev Behav Pediatr 27. https://doi.org/10.1097/00004703200604002-00013.

5. Howlin P, Charman T. Autism Spectrum Disorders: Interventions and Outcome. In: The SAGE Handbook of Developmental Disorders. 1 Oliver's Yard, 55 City Road, London EC1Y 1SP United Kingdom. SAGE Publications Ltd; 2011. pp. 307-28.

6. Emerson RW, Adams C, Nishino T, Hazlett HC, Wolff JJ, Zwaigenbaum L, et al. (2017): Functional neuroimaging of high-risk 6-month-old infants predicts a diagnosis of autism at 24 months of age. Sci Transl Med 9. https://doi.org/10.1126/scitranslmed.aag2882.

7. Cody Hazlett H, Gu H, Munsell BC, Hyung Kim S, Styner M, Wolff JJ, et al. Early brain development in infants at high risk for autism spectrum disorder HHS Public Access. Nature. 2017;542:348-51.

8. Tierney AL, Gabard-Durnam L, Vogel-Farley V, Tager-Flusberg H, Nelson CA. (2012): Developmental trajectories of resting eeg power: An endophenotype of autism spectrum disorder. PLoS ONE 7. https://doi.org/10.1371/journal.pone.0039127.

9. Wilkinson CL, Levin AR, Gabard-Durnam LJ, Tager-Flusberg H, Nelson CA. Reduced frontal gamma power at 24 months is associated with better expressive language in toddlers at risk for autism. Autism Res. 2019;12:1211-24.

10. Gabard-Durnam L, Tierney AL, Vogel-Farley V, Tager-Flusberg H, Nelson CA. Alpha asymmetry in infants at risk for autism spectrum disorders. J Autism Dev Disord. 2015;45:473-80.

11. Orekhova E, Elsabbagh M, Jones EJ, Dawson G, Charman T, Baron-Cohen S, et al. EEG hyperconnectivity in high-risk infants is associated with later autism. Journal of Neurodevelopmental Disorders. 2014;6:40. 
12. Righi G, Tierney AL, Tager-Flusberg H, Nelson CA. (2014): Functional connectivity in the first year of life in infants at risk for autism spectrum disorder: An EEG study. PLoS ONE 9. https://doi.org/10.1371/journal.pone.0105176.

13. Gabard-Durnam LJ, Wilkinson C, Kapur K, Tager-Flusberg H, Levin AR, Nelson CA. (2019): Longitudinal EEG power in the first postnatal year differentiates autism outcomes. Nat Commun 10. https://doi.org/10.1038/s41467-019-12202-9.

14. BosI W, Tierney A, Tager-Flusberg H, Nelson C. EEG complexity as a biomarker for autism spectrum disorder risk. BMC Med. 2011;9:18.

15. BosI WJ, Tager-Flusberg H, Nelson CA. (2018): EEG Analytics for Early Detection of Autism Spectrum Disorder: A data-driven approach. Sci Rep 8. https://doi.org/10.1038/s41598-018-24318-x.

16. Dickinson A, Daniel M, Marin A, Gaonkar B, Dapretto M, McDonald NM, Jeste S. Multivariate Neural Connectivity Patterns in Early Infancy Predict Later Autism Symptoms. Biological Psychiatry: Cognitive Neuroscience Neuroimaging. 2021;6:59-69.

17. Billstedt E, Gillberg C, Gillberg C. Autism after adolescence: Population-based 13- to 22-year follow-up study of 120 individuals with autism diagnosed in childhood. J Autism Dev Disord. 2005;35:351-60.

18. Magiati I, Tay XW, Howlin P. (2014): Cognitive, language, social and behavioural outcomes in adults with autism spectrum disorders: A systematic review of longitudinal follow-up studies in adulthood. Clinical Psychology Review, vol. 34. Elsevier Inc., pp 78-86.

19. Chawarska K, Klin A, Paul R, Macari S, Volkmar F. A prospective study of toddlers with ASD: Shortterm diagnostic and cognitive outcomes. J Child Psychol Psychiatry. 2009;50:1235-45.

20. Howlin P, Mawhood L, Rutter M. Autism and Developmental Receptive Language Disorder-a Followup Comparison in Early Adult Life. II: Social, Behavioural, and Psychiatric Outcomes. J Child Psychol Psychiatry. 2000;41:561-78.

21. Anderson DK, Lord C, Risi S, DiLavore PS, Shulman C, Thurm A, et al. Patterns of Growth in Verbal Abilities Among Children With Autism Spectrum Disorder. J Consult Clin Psychol. 2007;75:594-604.

22. Berger JM, Rohn TT, Oxford JT. (2012): Autism as the Early Closure of a Neuroplastic Critical Period Normally Seen in Adolescence. Biological Systems: Open Access 02. https://doi.org/10.4172/23296577.1000118.

23. Gao R, Penzes P. Common Mechanisms of Excitatory and Inhibitory Imbalance in Schizophrenia and Autism Spectrum Disorders. Curr Mol Med. 2015;15:146-67.

24. Ferri R, Elia M, Agarwal N, Lanuzza B, Musumeci SA, Pennisi G. The mismatch negativity and the P3a components of the auditory event-related potentials in autistic low-functioning subjects. Clin Neurophysiol. 2003;114:1671-80.

25. Martineau J, Garreau B, Roux S, Lelord G. Auditory evoked responses and their modifications during conditioning paradigm in autistic children. J Autism Dev Disord. 1987;17:525-39.

26. Bidet-Caulet A, Latinus M, Roux S, Malvy J, Bonnet-Brilhault F, Bruneau N. Atypical sound discrimination in children with ASD as indicated by cortical ERPs. Journal of Neurodevelopmental Disorders. 2017;9:13. 
27. Werker JF, Lalonde CE. (1988): Cross-Language Speech Perception: Initial Capabilities and Developmental Change. Developmental Psychology, vol. 24.

28. Minagawa-Kawai Y, Naoi N, Kikuchi N, Yamamoto JI, Nakamura K, Kojima S. Cerebral laterality for phonemic and prosodic cue decoding in children with autism. NeuroReport. 2009;20:1219-24.

29. Seery AM, Vogel-Farley V, Tager-Flusberg H, Nelson CA. Atypical lateralization of ERP response to native and non-native speech in infants at risk for autism spectrum disorder. Dev Cogn Neurosci. 2013;5:10-24.

30. Fagiolini M, Leblanc JJ. (2011): Autism: A critical period disorder? Neural Plasticity, vol. 2011. Hindawi Publishing Corporation. https://doi.org/10.1155/2011/921680.

31. Polka L, Werker JF. Developmental Changes in Perception of Nonnative Vowel Contrasts. J Exp Psychol Hum Percept Perform. 1994;20:421-35.

32. Hosseinifard B, Moradi MH, Rostami R. Classifying depression patients and normal subjects using machine learning techniques and nonlinear features from EEG signal. Comput Methods Programs Biomed. 2013;109:339-45.

33. Acharya UR, Sudarshan VK, Adeli H, Santhosh J, Koh JEW, Puthankatti SD, Adeli A. A novel depression diagnosis index using nonlinear features in EEG signals. Eur Neurol. 2015;74:79-83.

34. Bachmann M, Päeske L, Kalev K, Aarma K, Lehtmets A, Ööpik P, et al. Methods for classifying depression in single channel EEG using linear and nonlinear signal analysis. Comput Methods Programs Biomed. 2018;155:11-7.

35. Sabeti M, Katebi S, Boostani R. Entropy and complexity measures for EEG signal classification of schizophrenic and control participants. Artif Intell Med. 2009;47:263-74.

36. Boostani R, Sadatnezhad K, Sabeti M. An efficient classifier to diagnose of schizophrenia based on the EEG signals. Expert Syst Appl. 2009;36:6492-9.

37. Zhao Q, Hu B, Liu L, Ratcliffe M, Peng H, Zhai J, et al. (2012): An EEG based nonlinearity analysis method for schizophrenia diagnosis. Proceedings of the 9th IASTED International Conference on Biomedical Engineering, BioMed 2012136-142.

38. BosI WJ, Loddenkemper T, Nelson CA. Nonlinear EEG biomarker profiles for autism and absence epilepsy. Neuropsychiatric Electrophysiology. 2017;3:1.

39. Sathyanarayana A, el Atrache R, Jackson M, Alter AS, Mandl KD, Loddenkemper T, BosI WJ. Nonlinear Analysis of Visually Normal EEGs to Differentiate Benign Childhood Epilepsy with Centrotemporal Spikes (BECTS). Sci Rep. 2020;10:1-12.

40. Fergus $P$, Hussain A, Hignett D, Al-Jumeily D, Abdel-Aziz K, Hamdan H. A machine learning system for automated whole-brain seizure detection. Applied Computing Informatics. 2016;12:70-89.

41. Gabard-Durnam LJ, Mendez Leal AS, Wilkinson CL, Levin AR. The Harvard Automated Processing Pipeline for Electroencephalography (HAPPE): Standardized Processing Software for Developmental and High-Artifact Data. Frontiers in Neuroscience. 2018;12:97. 
42. Levin AR, Leal ASM, Gabard-Durnam LJ, O'Leary HM. (2018): BEAPP: The batch electroencephalography automated processing platform. Frontiers in Neuroscience 12. https://doi.org/10.3389/fnins.2018.00513.

43. Winkler I, Brandl S, Horn F, Waldburger E, Allefeld C, Tangermann M. (2014): Robust artifactual independent component classification for $\mathrm{BCl}$ practitioners. J Neural Eng 11. https://doi.org/10.1088/1741-2560/11/3/035013.

44. Levin AR, Varcin KJ, O'Leary HM, Tager-Flusberg H, Nelson CA. EEG power at 3 months in infants at high familial risk for autism. Journal of Neurodevelopmental Disorders. 2017;9:34.

45. NITRC: CleanLine. Tool/Resource Info (n.d.): Retrieved February 6, 2021, from https://www.nitrc.org/projects/cleanline.

46. Ortiz-Mantilla S, Hämäläinen JA, Realpe-Bonilla T, Benasich AA. Oscillatory dynamics underlying perceptual narrowing of native phoneme mapping from 6 to 12 months of age. J Neurosci. 2016;36:12095-105.

47. Cantiani C, Piazza C, Mornati G, Molteni M, Riva V. Oscillatory gamma activity mediates the pathway from socioeconomic status to language acquisition in infancy. Infant Behavior Development. 2019;57:101384.

48. Musacchia G, Ortiz-Mantilla S, Choudhury N, Realpe-Bonilla T, Roesler C, Benasich AA. Active auditory experience in infancy promotes brain plasticity in Theta and Gamma oscillations. Dev Cogn Neurosci. 2017;26:9-19.

49. Dehaene-Lambertz G. The human infant brain: A neural architecture able to learn language. Psychonomic Bulletin Review. 2017;24:48-55.

50. Sket GM, Overfeld J, Styner M, Gilmore JH, Entringer S, Wadhwa PD, et al. (2019): Neonatal White Matter Maturation Is Associated With Infant Language Development. Frontiers in Human Neuroscience 13. https://doi.org/10.3389/fnhum.2019.00434.

51. Bidet-Caulet A, Latinus M, Roux S, Malvy J, Bonnet-Brilhault F, Bruneau N. Atypical sound discrimination in children with ASD as indicated by cortical ERPs. Journal of Neurodevelopmental Disorders. 2017;9:13.

52. Lai G, Schneider HD, Schwarzenberger JC, Hirsch J. Speech stimulation during functional MR imaging as a potential indicator of autism. Radiology. 2011;260:521-30.

53. Liu J, Tsang T, Jackson L, Ponting C, Jeste SS, Bookheimer SY, Dapretto M. (2019): Altered lateralization of dorsal language tracts in 6-week-old infants at risk for autism. Developmental Science 22. https://doi.org/10.1111/desc.12768.

54. Gou Z, Choudhury N, Benasich AA. Resting frontal gamma power at 16, 24 and 36 months predicts individual differences in language and cognition at 4 and 5 years. Behav Brain Res. 2011;220:26370.

55. Tarullo AR, Obradović J, Keehn B, Rasheed MA, Siyal S, Nelson CA, Yousafzai AK. Gamma power in rural Pakistani children: Links to executive function and verbal ability. Dev Cogn Neurosci. 2017;26:1-8. 
56. Tomalski P, Moore DG, Ribeiro H, Axelsson EL, Murphy E, Karmiloff-Smith A, et al. Socioeconomic status and functional brain development - associations in early infancy. Developmental Science. 2013;16:676-87.

57. Benasich AA, Gou Z, Choudhury N, Harris KD. Early cognitive and language skills are linked to resting frontal gamma power across the first 3 years. Behav Brain Res. 2008;195:215-22.

58. Cantiani C, Ortiz-Mantilla S, Riva V, Piazza C, Bettoni R, Musacchia G, et al. (2019): Reduced leftlateralized pattern of event-related EEG oscillations in infants at familial risk for language and learning impairment. Neurolmage: Clinical 22. https://doi.org/10.1016/j.nicl.2019.101778.

59. Kolesnik A, Begum Ali J, Gliga T, Guiraud J, Charman T, Johnson MH, Jones EJH. Increased cortical reactivity to repeated tones at 8 months in infants with later ASD. Translational Psychiatry. 2019;9:1-11.

60. Arbabshirani MR, Plis S, Sui J, Calhoun VD. Single subject prediction of brain disorders in neuroimaging: Promises and pitfalls. Neurolmage. 2017;145:137-65.

61. Varoquaux G. (2018, October 15): Cross-validation failure: Small sample sizes lead to large error bars. Neurolmage, vol. 180. Academic Press Inc., pp 68-77.

62. Schnack HG, Kahn RS. (2016): Detecting neuroimaging biomarkers for psychiatric disorders: Sample size matters. Front Psychiatry 7. https://doi.org/10.3389/fpsyt.2016.00050.

\section{Tables}

Due to technical limitations, the tables are only available as a download in the supplemental files section.

\section{Figures}



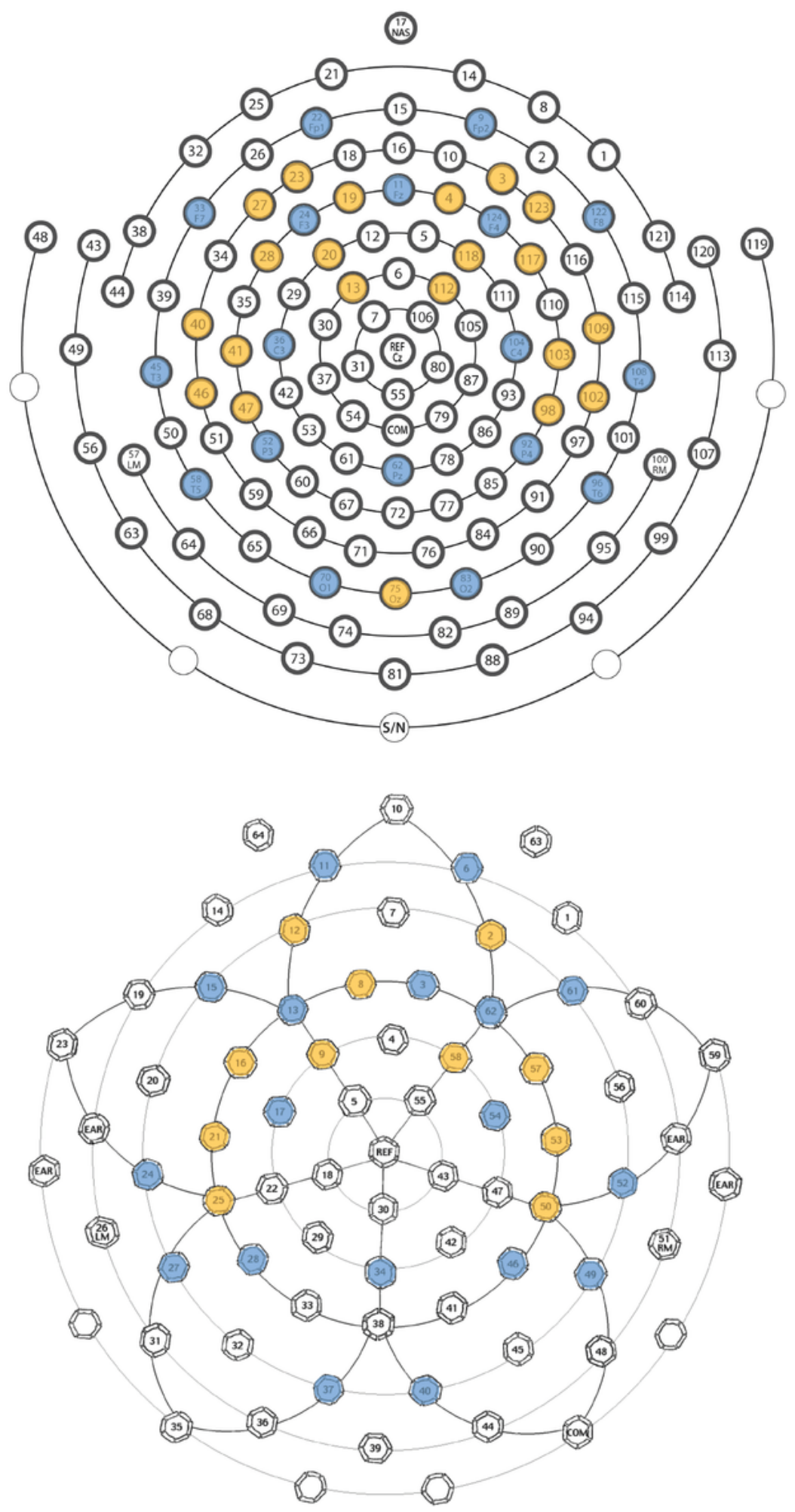

\section{Figure 1}

EEG net layouts. Two EEG nets were used in the study: the 128-channel EGI HydroCel Geodesic Sensor Net (version 1.0) presented on the top and the 64-channel EGI Geodesic Sensor Net (version 2.0) presented on the bottom. The 10-20 montage channels evaluated in this study are highlighted in blue, and HAPPE channels included in preprocessing steps are highlighted in yellow. 

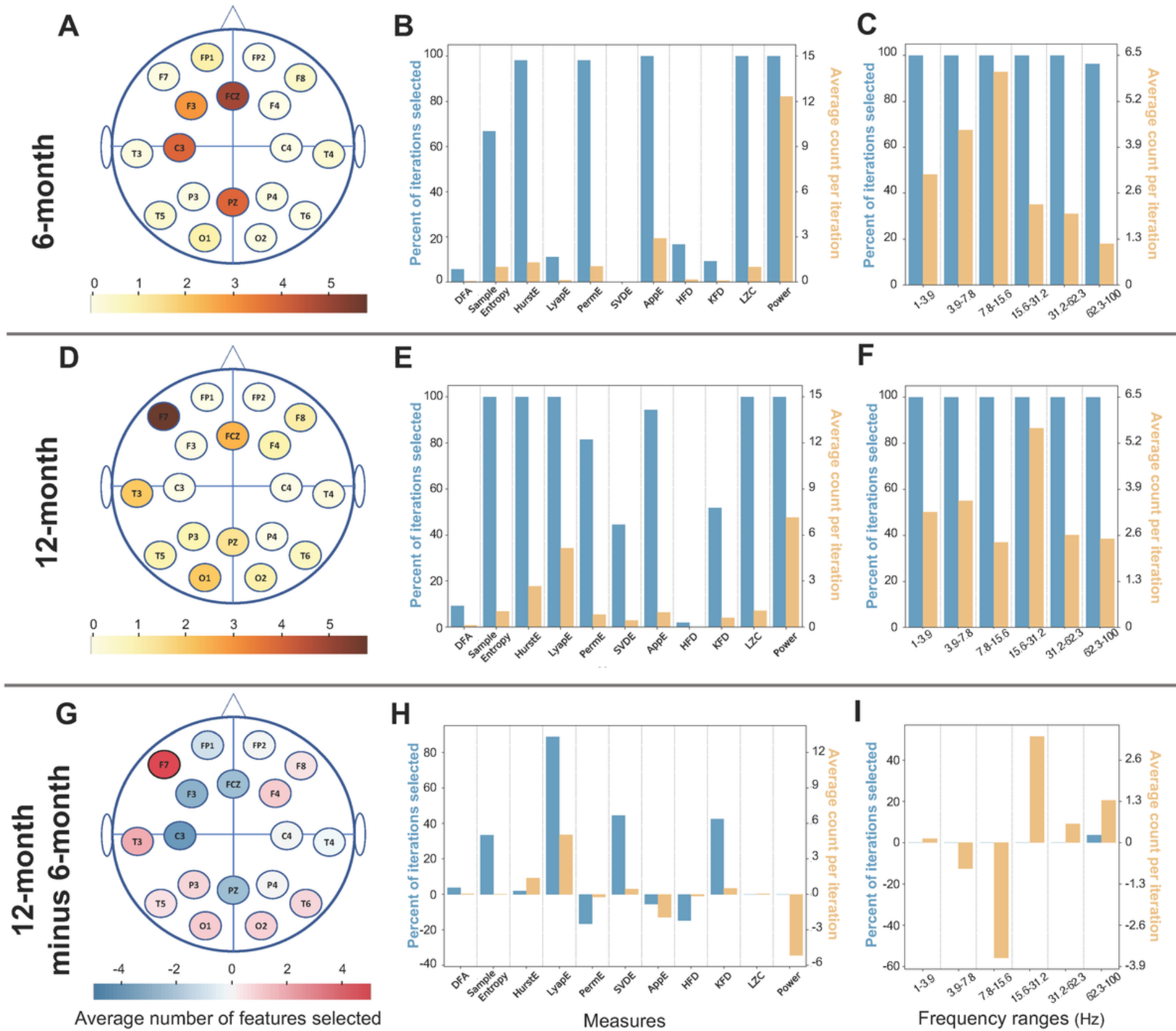

Figure 2

Information about features most correlated with autism diagnostic outcome for nearly overlapping 6-and 12-month analyses $(n=54)$. The bottom row visualizes the values for the 12-month dataset (middle row) minus the 6-month dataset (top row). (A, D, G) Average number of features selected from each channel. Color indicates number of features selected from a given channel. $(B, E, H)$ Average count of each EEG measure across iterations (orange) and percentage of iterations that each measure was selected at least once (blue). (C, F, I) Average count of each wavelet across iterations (orange) and percentage of iterations that each wavelet was selected at least once (blue).

\section{Supplementary Files}


This is a list of supplementary files associated with this preprint. Click to download.

- PeckJNDDSupplmental.pdf

- drawingimage4.png

- drawingimage3.png

- drawingimage2.png

- drawingimage1.png 